\title{
Assessment of cardiac indices using M-mode echocardiography in horse with tachycardia
}

\author{
Mohamed Ahmed Youssef, Medhat Nasef Mohamed, Sabry Ahmed EL-Khodery, Maged Rezk EL-Ashker, Alshimaa Mohammed \\ Mohammed Ali Farag
}

Department of Internal Medicine, Infectious Diseases and Fish Diseases, Faculty of Veterinary Medicine, Mansoura University, Mansoura, 35516, Egypt

\begin{abstract}
The aim of this study was to assess the effect of tachycardia associated with abdominal pain in horse with colic on echocardiographic measurements. For this purpose, 20 horses were selected for this study; from which 10 healthy horses were randomly selected (control group), and 10 diseased horses exhibiting the clinical signs of flatulent colic referred to the hospital of Faculty of Veterinary Medicine, Mansoura University, Egypt. Cardiac functions were evaluated using $\mathrm{M}$ - mode echocardiography for measurement of the cardiac indices and functions. In horses with flatulent colic, there was a significant $(P<0.05)$ decrease in interventricular septal thickness at end- systole (IVSTs), left ventricular internal diameter at end- diastole (LVIDd), left ventricular posterior wall at end-diastole (LVPWd), stroke volume (SV), and ejection fraction (EF) compared with control group. However, there was a significant $(P<0.05)$ increase in left ventricular volume at end- systole (ESV) in comparison with control group. The results of the present study indicate that evaluation of cardiac indices and functions via echocardiography may be of great benefit for evaluation of clinical outcome of tachycardia associated with abdominal pain in horses.
\end{abstract}

Keywords: M-mode; Echocardiography; cardiac indices; cardiac functions; flatulent colic; healthy horses; diseased horses.

\section{INTRODUCTION}

The "Heart Rate (HR) and Heart Rate Variability (HRV)" has taken together experts in the field of animal and veterinary sciences who are concerned with research on cardiac activity and HRV in farm animals (von Borell et al., 2007). Heart rate, at any time point in healthy individuals, represents the net interaction between vagal (which reduces $H R$ ) and sympathetic (which increases $H R$ ) regulation (Friedman and Thayer, 1998). However, the interaction between the branches is relatively complex, meaning that the heart rate parameters provide data on the net effects of all components inputting into cardiac activity and are of limited use for accurately assessing sympatho-vagal regulation (Tulppo et al., 1998).

Heart rate variability is defined as the complex beat-tobeat variation in HR produced by the interaction between sympathetic and parasympathetic (vagal) neural activity at the sinus node of the heart (Thayer and Sternberg, 2006; von Borell et al., 2007). Thus, analysis of the HRV allows a much more accurate and detailed determination of the functional regulatory characteristics of the autonomic nervous system (Taylor and Lipsitz, 1997). From the clinical point of view, HRV is used to assess the stress reaction in horses in response to aggressive handling, inappropriate stabling, feeding, or longdistance traveling as well as stress from pain or illness (
Rietmann et al., 2004 ;Dujardin and van Loon, 2011). Both, the physiological stress response and the behavioral signs of severe and acute pain are well described in horses to induce elevated HR, respiratory rate, body temperature and blood pressure (Rietmann et al., 2004). Respiratory rate and HR have been extensively studied in relation to abdominal pain by equine veterinarians as an important indicator of pain and the need for analgesia. However, these parameters are nonspecific for the presence as well as assessment of severity of pain, but also influenced by other factors, including dehydration, excitement and cardiovascular and/or respiratory diseases (Dujardin and van Loon, 2011).

Pain originating from the anterior portion of the intestinal tract (stomach and small intestine) is usually more severe other than pain arising from the cecum and colon. Obstructive lesion (volvulus, torsion, and intussusception), impaction lesion resulted in a similar severe type of pain. However, spasmodic colic aroused from indigestion or minor thromboembolic episodes resulted in intermittent type of pain. While it is important to control the pain and prevent the animal from injuring itself or its handlers, one should try to avoid masking the pain completely before making some kind of decision as to its source (Ashley et al., 2005 ). Studies have often failed to establish a direct relation between $H R$ and the presence or severity of pain (Dzikiti et al., 2003). 
In clinical practice, equine pain scoring system is the gold standard approach for the determination of the absence, presence, and intensity of pain, such as (visual analogue scales, simple descriptive scales, numerical rating scales, time budget analysis, composite pain scales and grimace scales) and highlights their strength and weakness for potential clinical implementation (De Grauw and van Loon, 2016). While equine pain scoring system provides important clinical reference indicators and proves to be a valid method for the adequate therapy of animals suffered from pain in most situations, it would fail to be applied in certain diseased condition (De Grauw and van Loon, 2016). More recently, abdominal radiography and abdominal ultrasonography have become more useful in evaluating horses with abdominal pain (Klohnen, 2012). Few studies have assessed the automatic recognition of pain intensity from physiological signals through electrocardiography (ECG) which is considered the gold standard for definitively diagnosing rhythm disorders and permits monitoring of the horses' HR and rhythm during exercise (Menzies-Gow, 2001). Moreover, echocardiography allows subjective and objective assessment of cardiac chamber dimensions and serves to describe and quantify the left ventricle systolic function characteristics (Sabev, 2014). Therefore, the aim of the present study is to assess the effect of severe acute abdominal pain on the cardiac function and indices in horses with flatulent colic using M-mode echocardiography.

\section{MATERIALS AND METHODS}

\section{Animals}

Ten horses exhibiting the clinical signs of flatulent colic referred to the hospital of Faculty of Veterinary Medicine, Mansoura University, Mansoura, Egypt between September 2017 and September 2018 were included in the study. In addition, ten healthy horses at the same age and under the same nutritional and environmental condition were randomly selected as a control group. This study was approved by the Animal Welfare and Ethics Committee, Mansoura University, Egypt on October, 2017.

\section{Clinical examinations}

A detailed clinical examination of the horses was carried out immediately after arrival in the hospital according to a standard protocol [15] and clinical findings were recorded including rectal temperature 36.7 - 38.5 으, heart rate 85 100 beat/minute, respiratory rate $11-32$ respiratory cycle/minute, congested color mucous membranes, CRT > 2 seconds, obvious abdominal distension with acute severe abdominal pain, and absence of the auscultable peristaltic sounds. On rectal examination, gas-filled loops of the large intestine were felt inside the abdominal cavity and the proper examination of its contents is impossible. Moreover, using stomach tube, there is no any reflux of gastro-intestinal content.

\section{Echocardiographic procedures}

Transcutaneous echocardiographic examinations were performed according to the standard methods described by (Youssef et al., 2016). All echocardiographic procedures and precaution were followed according to the recommendations of the American Society of Echocardiography. The M-mode echocardiography were performed with a CHISON Digital Color Doppler Ultrasound System, iVis 60 EXPERT VET, CHISON Medical Imaging Co., Ltd, China), using 2 - 3.9 MHz phased array transducer, with a maximal depth of $24.1 \mathrm{~cm}$. For guided M-mode measurements, a left parasternal short-axis of left ventricle with papillary muscles view was used. It was obtained by rotating the transducer through $90^{\circ}$ in clockwise direction with a slight cranial and/or dorsal angulation. Location of the axial beam was through the left ventricle at the level of chordae tendineae of the mitral valve and the junction of the left ventricular wall and the interventricular septum. Measurement of echocardiographic parameters, including interventricular septal thickness at end-systole (IVSTs) and at end-diastole (IVSTd), left ventricular internal diameter at end-systole (LVIDs) and at end-diastole (LVIDd), left ventricular posterior wall thickness at end-systole (LVPWs) and at end-diastole (LVPWd) were performed through Cube Method (Reef, 1998; Michima et al., 2004).

Standardized image planes were obtained by B-mode which was used to guide M-mode views for the measurements. Through Teicholz Method, left ventricular volume at end-diastole (EDV) and at end-systole (ESV) were calculated (Hanton et al., 1998) then the stroke volume was calculated from the following equation, Stroke volume = EDVESV. The left ventricular M-mode measurements were used to calculate the fractional shortening (FS \%) and ejection fraction (EF \%) (Kienle and Thomas, 2002).

\section{Data analysis}

Data were subjected to statistical analysis using statistical software program (SPSS for Windows, version 21, USA). Data were analyzed for normal distribution using KolmogorovSmirnov normality test. Data were normally distributed; therefore, mean and standard deviation for each variable were statistically analyzed and presented. Independent sample $t$-test was used to assess statistical differences between the two groups. For all statistical examinations, results were considered significant at $\mathrm{P}<0.05$.

\section{RESULTS}

Echocardiographic measurements from the left parasternal short-axis left ventricle with papillary muscles 
view, showed IVS, LVID, and LVPW at end-diastole and at endsystole in healthy and diseased horses, using $\mathrm{M}$-mode echocardiography (Figure 2.1).

In horses with flatulent colic compared to control group, there was a significant $(P<0.05)$ decrease in IVSTs $(\mathrm{cm})(2.1 \pm$ 0.6 vs. $4.4 \pm 0.3)$, LVIDd (cm) $(6.4 \pm 1.9$ vs. $6.6 \pm 0.7)$, LVPWd (cm) $(2.2 \pm 0.4$ vs. $3.3 \pm 1.1)$, SV (ml) $(70.8 \pm 28.9$ vs. $197.7 \pm$ 24.3), and $\operatorname{EF}(\%)(58.8 \pm 11.8$ vs. $90.3 \pm 2.8)$. However, there was a significant $(P<0.05)$ increase in ESV $(\mathrm{ml})(97.9 \pm 16.2$ vs. $28.3 \pm 15.9$ ) in comparison with control group (Table 2.1).

Table 1. Cardiac Indices (mean values \pm SD) in Horses assessed by M-mode Echocardiography

\begin{tabular}{|c|c|c|c|}
\hline $\begin{array}{l}\text { Cardiac } \\
\text { Indices }\end{array}$ & $\begin{array}{l}\text { Control } \\
\text { group }\end{array}$ & $\begin{array}{l}\text { Diseased } \\
\text { group }\end{array}$ & P-value \\
\hline IVSTd (cm) & $2.7 \pm 0.2$ & $1.7 \pm 0.5$ & 0.065 \\
\hline IVSTs (cm) & $4.4 \pm 0.3$ & $2.1 \pm 0.6^{*}$ & 0.010 \\
\hline LVIDd (cm) & $6.6 \pm 0.7$ & $6.4 \pm 1.9^{*}$ & 0.044 \\
\hline LVIDs (cm) & $3.1 \pm 0.5$ & $4.6 \pm 1.3$ & 0.078 \\
\hline LVPWd (cm) & $3.3 \pm 1.1$ & $2.2 \pm 0.4^{*}$ & 0.010 \\
\hline LVPWs (cm) & $3.9 \pm 0.7$ & $2.6 \pm 0.7$ & 0.818 \\
\hline EDV (ml) & $216.2 \pm 27.3$ & $271.1 \pm 72.4$ & 0.206 \\
\hline ESV (ml) & $28.3 \pm 15.9$ & $97.9 \pm 16.2^{* *}$ & 0.001 \\
\hline SV (ml) & $197.7 \pm 24.3$ & $70.8 \pm 28.9^{* *}$ & 0.001 \\
\hline EF $(\%)$ & $90.3 \pm 2.8$ & $58.8 \pm 11.8^{*}$ & 0.041 \\
\hline FS (\%) & $54.3 \pm 4.1$ & $26.5 \pm 7.2$ & 0.335 \\
\hline \multicolumn{4}{|c|}{$\begin{array}{l}\text { IVSTd : interventricular septal thickness at end -diastole ; IVSTs : } \\
\text { interventricular septal thickness at end-systole; LVIDd : left ventricular } \\
\text { internal diameter at end -diastole; LVIDs : left ventricular internal } \\
\text { diameter at end-systole; LVPWd : left ventricular posterior wall } \\
\text { thickness at end-diastole; LVPWs : left ventricular posterior wall } \\
\text { thickness at end- systole; EDV : left ventricular volume at end-diastole; } \\
\text { ESV : left ventricular volume at end-systole; SV :stroke volume; EF : } \\
\text { ejection fraction; and FS : fractional shortening. }\end{array}$} \\
\hline
\end{tabular}

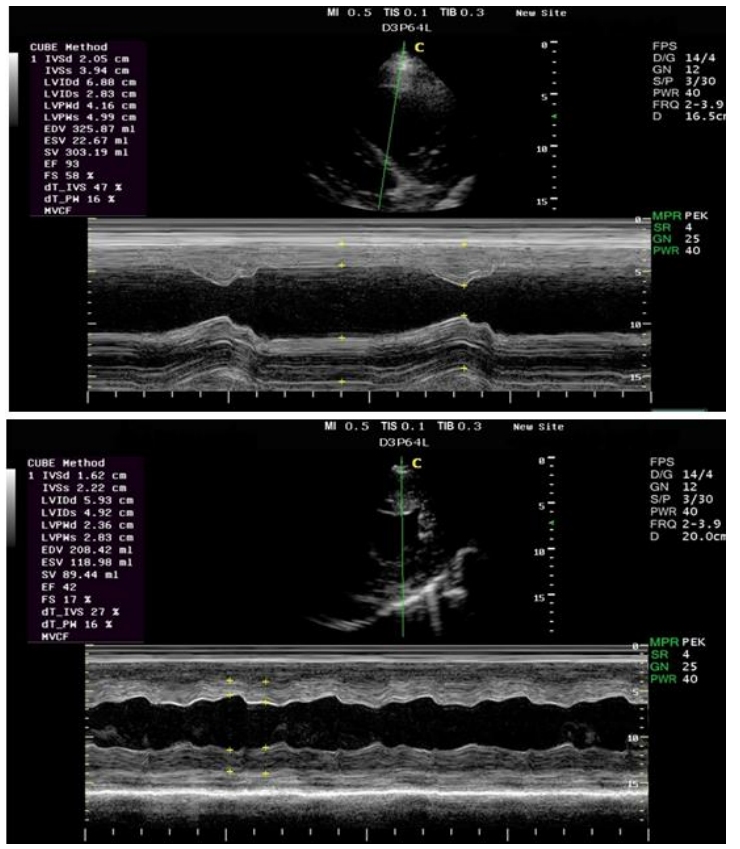

Figure 2.1 (a) The left parasternal short-axis left ventricle with papillary muscles view in healthy horses. (b) The left parasternal short-axis view of the heart in diseased horses, during diastole, and during systole using $\mathrm{M}$-mode echocardiography. IVS= interventricular septum, LVID= left ventricular internal diameter, LVPW $=$ left ventricular posterior wall.

\section{DISCUSSION}

Abdominal pain in horses is one of the most common presenting clinical signs and a major cause of mortality (Klohnen, 2012). Although heart rate may vary in animals in pain, the change in heart rate caused by other causes such as shock and endotoxemia may have an unknown influence on the HR under these conditions (Pascoe et al., 1990); hence, it can only be taken as a measure of the intensity of pain in the context of having removed or accounted for other possible reasons for the observed change (Taylor et al., 2002). The validation of a change in HR as a measure of pain may also depend on the use of some other behavioral test or observation that may be subjective (Taylor et al., 2002).Therefore, the objective of this study was to use Mmode echocardiography to assess left ventricle systolic function and cardiac contractility, which precisely reflect the HRV in horses.

The echocardiographic parameters of the left ventricle, including chamber size and wall thicknesses are used to diagnose and to assess the severity of valvular regurgitation (Reef, 1998), dilated cardiomyopathy (Hughes et al., 2009), and to predict the athletic performance (Buhl et al., 2005) in equines. Transient changes in left ventricular geometry occur as a result of reduced left ventricular volume (Di Segni et al., 
1997), with aresultant what is called pseudohypertrophy (Duvekot et al., 1994; Di Segni et al., 1997; Campbell and Kittleson, 2007; Fine et al., 2010), which is evidenced by reduced left ventricular internal diameter (LVID), and increased the ventricular wall and septal thicknesses during diastole.

In horses with flatulent colic, the IVSs, LVIDd, and LVPWd were significantly $(P<0.05)$ decreased indicating a decrease in preload. Such results may be due to pain and dehydration which induced alterations in the cardiac function with decreased in diastolic echocardiographic measurements (Fine et al., 2010).

The left ventricle (LV) end systolic volume (ESV) was significantly increased in horses with flatulent colic in comparison with control group, suggesting left ventricle systolic dysfunction. These findings may be attributed to the changes occurring in the contractility of the heart. It has been proposed that ESV is affected by afterload and contractility of the heart (Aurigemma et al., 2002) . Moreover, afterload in the left side of the heart may be elevated in case of increased systemic pressure, vasoconstriction, and obstruction to ventricular outflow (Amory et al., 2010). On the other hand, David et al., (2008) suggested that ESV is less sensitive to cardiac loading and varies greatly in response to changes in contractility.

There was a significant $(P<0.05)$ decrease in both stroke volume and ejection fraction in horse with flatulent colic compared with control group. Such results may be attributed to the stress created by abdominal pain. Similarly, the greater contraction force of the heart is also required during a highly intense exercise (Bonomo et al., 2011). Stroke volume and ejection fraction support each other for evaluation of the heart. Where SV is used clinically as a measurement of the global heart function; it is a very insensitive indicator of cardiac function (Marr, 1994). Ejection fraction (EF) is commonly used clinically as a mean for assessment of the global LV function and risk stratifying animals with heart failure (Lightowler et al., 2000). EF is affected by extrinsic factors to the LV, thus decreasing its accuracy as a measure of cardiac function. Some have argued that, instead of reflecting myocardial contractility, EF is largely driven by the degree of LV dilatation (Young and Scott, 1998). Heart failure with reduced ejection fraction may be found in animals with thyrotoxicosis in spite of the fact that it is a hyper metabolic condition. Some predisposing conditions include pre-existing hypertension, ischemic heart disease, and mitral valve disease, which may be associated with heart failure (Ikram, 1985). Consequently, this parameter may be not confirmative for diagnosis of abdominal pain only. Reduced ejection fraction is usually caused by direct cardiac damage due to autoimmune myocarditis, and congestive circulation (Bond et al., 1988). Animals with reduced ejection fraction due to systolic dysfunction and tachycardia will respond to treatments aimed at slowing the heart rate or controlling the ventricular response in atrial fibrillation (Levey and Klein, 1990; Buhl et al., 2007).

\section{Conclusion}

The cardiac indices using M-mode echocardiography in horse with tachycardia are changed including interventricular septal thickness at end- systole (IVSTs), left ventricular internal diameter at end - diastole ( LVIDd), left ventricular posterior wall thickness at end-diastole (LVPWd), stroke volume (SV), left ventricular volume at end-systole (ESV), and ejection fractional percentage (EF \%), with a resultant alteration in the cardiac functions.

\section{REFERENCES}

Amory, H., Votion, D.-M., Fraipont, A., Goachet, A.G., Robert, C., Farnir, F. Van Erck, E., 2010. Altered Systolic Left Ventricular Function In Horses Completing A Long Distance Endurance Race. Equine Veterinary Journal, 42, 216-219.

Ashley, F.H., Waterman-Pearson, A.E., Whay, H.R., 2005. Behavioural assessment of pain in horses and donkeys: application to clinical practice and future studies. Equine Veterinary Journal, 37, 565-575.

Aurigemma G, Douglas, P., Gaasch, W. , 2002. Quantitative evaluation of left ventricular structure, wall stress, and systolic function. In: Otto $\mathrm{C}$, ed. The practice of Clinical Echocardiography. 2nd ed. Philadelphia: WB Saunders Company, pp 65-87.

Bond, B.R., Fox, P.R., Peterson, M.E., Skavaril, R.V., 1988. Echocardiographic findings in 103 cats with hyperthyroidism. Journal of the American Veterinary Medical Association, 192, 1546-1549.

Bonomo, C.C.M., Mishima, L.E.S., Miyashiro, P., Fernandes, W.R., 2011. Quantitative Echocardiography of Athletic Quarter Horses Ars Veterinaria, Jaboticabal, SP 27, 220-225

Buhl, R., Ersbll, A.K., Eriksen, L., Koch, J.,2005 .Changes overtime in echocardiographic measurements in young Standardbred racehorses undergoing training and racing and association with racing performance. Journal of the American Veterinary Medical Association, 226, 1881-1887

Buhl, R., Ersboll, A.K., Larsen, N.H., Eriksen, L., Koch, J., 2007.The effects of detomidine, romifidine or acepromazine on echocardiographic measurements and cardiac function in normal horses. Veterinary Anaesthesia and Analgesia, 34, 1-8

Campbell, F.E., Kittleson, M.D., 2007. The effect of hydration status on the echocardiographic measurements of normal cats. Journal of Veterinary Internal Medicine, 21, 1008-1015.

David, D. M., Sanjiv, J. S., Mary, R. F., Alisa, R., Mary, A. W., Nelson, B. S., 2008. Prognostic Value of Left Ventricular End-Systolic Volume Index as a Predictor of Heart Failure Hospitalization in Stable Coronary Artery Disease: Data from the Heart and Soul Study. Journal of the American Society of Echocardiography. 22, 190-197.

De Grauw, J.C., van Loon, J.P., 2016. Systematic pain assessment in horses. Veterinary journal (London, England : 1997), 209, 14-22.

Di Segni, E., Preisman, S., Ohad, D.G., Battier, A., Boyko, V., Kaplinsky, E., Perel, A., Vered, Z., 1997. Echocardiographic left ventricular remodeling and pseudohypertrophy as markers of hypovolemia. An experimental study on bleeding and volume repletion. Journal of The American Society of Echocardiography, 10, 926-936.

Dujardin, C.L., van Loon, J.P., 2011. Pain recognition and treatment in the horse: A survey of equine veterinarians in The Netherlands and Belgium.Tijdschrift voor Diergeneeskunde, 136,715-724.

Duvekot, J.J., Cheriex, E.C., Tan, W.D., Heidendal, G.A., Peeters, L.L., 1994. Volume-dependent echocardiographic parameters are not useful for estimating baseline blood volume but are useful for detecting 
acute changes in vascular filling state. Basic Research in Cardiology, 89, 270-277.

Dzikiti, T. B., Hellebrekers, L. J., \& Dijk, P., 2003. Effects of Intravenous Lidocaine on Isoflurane Concentration, Physiological Parameters, Metabolic Parameters and Stress-related Hormones in Horses Undergoing Surgery. Journal of Veterinary Medicine Series A, 50, 190-195.

Fine, D.M., Durham, H.E., Durham Jr, H.E., Rossi, N.F., Spier, A.W., Selting, K., Rubin, L.J., 2010. Echocardiographic assessment of hemodynamic changes produced by two methods of inducing fluid deficit in dogs. Journal of Veterinary Internal Medicine, 24, 348-353.

Friedman, B.H., Thayer, J.F., 1998. Anxiety and autonomic flexibility: a cardiovascular approach. Biological Psychology, 49, 303-323.

Hanton, G., Geffray, B., Lodola, A., 1998. Echocardiography, non-invasive method for the investigation of heart morphology and function in laboratory dogs: 1-method and reference values for $\mathrm{M}$-mode parmeters. Laboratory Animals, Apr; 32, 173-182.

Hughes K.J., Hoffmann, K.L., Hodgson D.R., 2009. Long-term assessment of horses and ponies post exposure to monensin sodium in commercial feed. Equine Veterinary Journal, 41, 47-52.

Ikram, H., 1985. The nature and prognosis of thyrotoxic heart disease. The Quarterly Journal of Medicine, 54, 19-28.

Kienle, R.D., Thomas, W.D., 2002. Echocardiography.In: Small Animal Diagnostic Ultrasound, 2nd Edition.,Saunders, Philadelphia,U.S.A., 354-423.

Klohnen, A., 2012. Abdominal Ultrasonography In The Equine Patient With Acute Signs Of Colic. Ultrasound Of The Thorax And Abdomen, 58, 11-18.

Levey, G.S., Klein, I., 1990. Catecholamine-thyroid hormone interactions and the cardiovascular manifestations of hyperthyroidism. The American Journal of Medicine, 88, 642-646.

Lightowler, C.H., Pidal, G., Mercado, M., Cattaneo, M.L., 2000. Athlete's heart: a meta-analysis of the echocardiographic experience. Archiev Medical Veterinary, 32, 229-234.

Marr, C.M., 1994. Equine echocardiography--sound advice at the heart of the matter. The British Veterinary Journal, 150, 527-545.

Menzies-Gow, N., 2001. ECG interpretation in the horse. In Practice, 23, 454459.

Michima, L.E.S., Latorre, S.M., Andrade, A.F., Fernandes, W.R., , 2004. Bmode and M-mode echocardiography of endurance horses raised in sõo Paulo state ,Brazil, 24, 451-457.

Pascoe, P.J., Ducharme, N.G., Ducharme, G.R., Lumsden, J.H., 1990. A computer-derived protocol using recursive partitioning to aid in estimating prognosis of horses with abdominal pain in referral hospitals. Canadian Journal of Veterinary Research, 54, 373-378.

Reef, V., 1998 .Cardiovascular ultrasonography. In: Equine Diagnostic Ultrasound, pp. 215-272.

Rietmann, T.R., Stauffacher, M., Bernasconi, P., Auer, J.A., Weishaupt, M.A., 2004. The association between heart rate, heart rate variability, endocrine and behavioural pain measures in horses suffering from laminitis. Journal of Veterinary Medicine. A, Physiology, Pathology, Clinical Medicine, 51, 218-225.

Sabev, S.P., 2014. Cardiac structures measurements by echocardiography in clinically healthy warmblood horses. Bulgarian Journal of Veterinary Medicine,17, 267-275.

Taylor, J.A., Lipsitz, L.A.,1997. Heart rate variability standards. Circulation, 95, 280-281.

Taylor, P.M., Pascoe, P.J., Mama, K.R., 2002. Diagnosing and treating pain in the horse. Where are we today?. The Veterinary clinics of North America Equine practice, 18, 1-19.

Thayer, J.F., Sternberg, E., 2006. Beyond heart rate variability: vagal regulation of allostatic systems. Annals of the New York Academy of Sciences, 1088, 361-372.

Tulppo, M.P., Makikallio, T.H., Seppanen, T., Laukkanen, R.T., Huikuri, H.V., 1998. Vagal modulation of heart rate during exercise: effects of age and physical fitness. The American Journal of Physiology, 274, H424-429.

von Borell, E., Langbein, J., Despres, G., Hansen, S., Leterrier, C., MarchantForde, J., Marchant-Forde, R., Minero, M., Mohr, E., Prunier, A., Valance, D., Veissier, I., 2007. Heart rate variability as a measure of autonomic regulation of cardiac activity for assessing stress and welfare in farm animals -- a review. Physiology and Behavior, 92, 293-316.

Young, L.E., Scott, G.R., 1998. Measurement of cardiac function by transthoracic echocardiography: day to day variability and repeatability in normal Thoroughbred horses. Equine Veterinary Journal, 23, 117-122.

Youssef, M.A., Ibrahim, H.M., Farag, E.-S.M., El-Khodery, S.A., 2016. Effects of Tilmicosin Phosphate Administration on Echocardiographic Parameters in Healthy Donkeys (Equus asinus): An Experimental Study. Journal of Equine Veterinary Science, 38, 24-29.

Mans Vet Med J 20:1 (2019) 1-5 